\title{
Terutroban et récepteurs TP endothéliaux dans l'athérogenèse
}

> Le traitement des maladies thrombotiques implique l'utilisation de substances antiplaquettaires, anti-coagulantes ou profibrinolytiques. L'action antiplaquettaire de l'aspirine est due à l'inhibition indirecte de la production du thromboxane $A_{2}\left(T X A_{2}\right)$, un puissant vasoconstricteur également activateur des plaquettes. Le terutroban (S 18886), quant à lui, agit plus spécifiquement comme antagoniste sélectif des récepteurs TP, les récepteurs du TXA $A_{2}$ qui, dans le système vasculaire, se trouvent sur les plaquettes, les cellules musculaires lisses et l'endothélium. Les études précliniques et cliniques effectuées avec le terutroban ont démontré ses effets antithrombotiques. Le but de cette revue est de résumer le rôle des récepteurs TP endothéliaux dans l'athérogenèse et de décrire les études qui, menées avec le terutroban, ont contribué à établir ce rôle. En effet, l'activation des récepteurs TP endothéliaux, en provoquant l'expression de molécules d'adhérence qui favorisent l'adhérence et l'infiltration de monocytes/macrophages dans la paroi artérielle, participe à la genèse de l'athérosclérose. <

Le récepteur TP est le récepteur du thromboxane $A_{2}$ $\left(T X A_{2}\right)$, l'un des principaux métabolites de l'acide arachidonique. L'activation des récepteurs TP par le $\mathrm{TXA}_{2}$, ou par d'autres ligands, provoque l'agrégation des plaquettes, la vasoconstriction et la bronchoconstriction. Les récepteurs TP interviennent également dans la prolifération cellulaire, l'apoptose et l'angiogenèse.

Le terutroban (S 18886) est un antagoniste puissant et sélectif des récepteurs TP; actif par voie orale et présentant une longue durée d'action, il est actuellement en phase III de son développement clinique pour le traitement et la prévention des maladies cardiovasculaires.

Il a également permis, au cours de récentes expériences menées dans des modèles d'athérosclérose, de

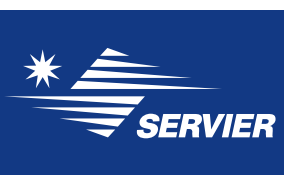

Division d'Angiologie,

Institut de Recherche Servier,

11 , rue des Moulineaux,

92150 Suresnes, France.

tony.verbeuren@

fr.netgrs.com

montrer l'importance des récepteurs TP endothéliaux dans les mécanismes d'adhérence cellulaire pathologique.

\section{De la découverte du TXA ${ }_{2}$ aux récepteurs TP}

En 1975, Samuelson et ses collaborateurs ont rapporté l'existence d'un métabolite instable de l'acide arachidonique, le thromboxane $A_{2}$, dérivé oxane produit par les plaquettes sanguines (les thrombocytes) [1]. L'enzyme responsable de sa synthèse est la thromboxane synthétase [2]. L'aspirine bloque la synthèse du TXA ${ }_{2}$ [2] en inhibant la cyclo-oxygénase (COX) (dont il existe deux isoformes, COX-1 et COX-2), une enzyme responsable de la formation des endoperoxydes de l'acide arachidonique [3].

Métabolite dominant de l'acide arachidonique (Figure 1) produit par les plaquettes activées, le TXA est considéré comme l'un des plus puissants activateurs des plaquettes et agents vasoconstricteurs. Un dérèglement de sa synthèse ou de son action est retrouvé dans de nombreuses maladies (maladies cardiovasculaires, asthme). L'aspirine, l'un des traitements majeurs dans la prévention des pathologies vasculaires coronaires et cérébrales, agit ainsi en inhibant la synthèse du TXA ${ }_{2}[3]$.

Le $\mathrm{TXA}_{2}$ se lie avec des récepteurs membranaires spécifiques (Figure 1 ), les récepteurs TP, qui appartiennent à la classe des récepteurs des prostanoïdes $(P)$ et possèdent une haute affinité pour le $\mathrm{TXA}_{2}(\mathrm{~T})$ [4]. Deux isoformes, TP $\alpha$ et TP $\beta$, existent chez I'homme, mais probablement pas chez les autres espèces; dérivées d'un épissage alternatif, elles sont identiques pour leurs 328 résidus aminoterminaux [4-6]. Le mode de signalisation majeur du récepteur TP 
est l'activation des $\beta$-isozymes de la phospholipase $C$, qui aboutit à un turnover de phosphatidylinositol et à la libération de calcium; au moins 9 protéines $G$ peuvent être impliquées. La différence principale entre les deux isoformes des récepteurs TP siège dans la modulation de l'adénylyl cyclase, activée par TP $\alpha$ mais inhibée par TP $\beta$ [7].

La caractérisation moléculaire et fonctionnelle complète des récepteurs TP nécessite encore des études complémentaires.

\section{Le terutroban ( $S$ 18886), un antagoniste sélectif des récepteurs TP}

Une fois l'importance du TXA ${ }_{2}$ comme vasoconstricteur puissant et activateur des plaquettes reconnue, et le lien entre l'action de l'aspirine et l'inhibition de la synthèse du TXA ${ }_{2}$ établi, la recherche de composés capables d'inhiber de façon plus spécifique la synthèse ou l'action du TXA 2 a débuté.

Les inhibiteurs de la thromboxane synthétase (TSI) (Figure 1) ont montré une activité assez décevante

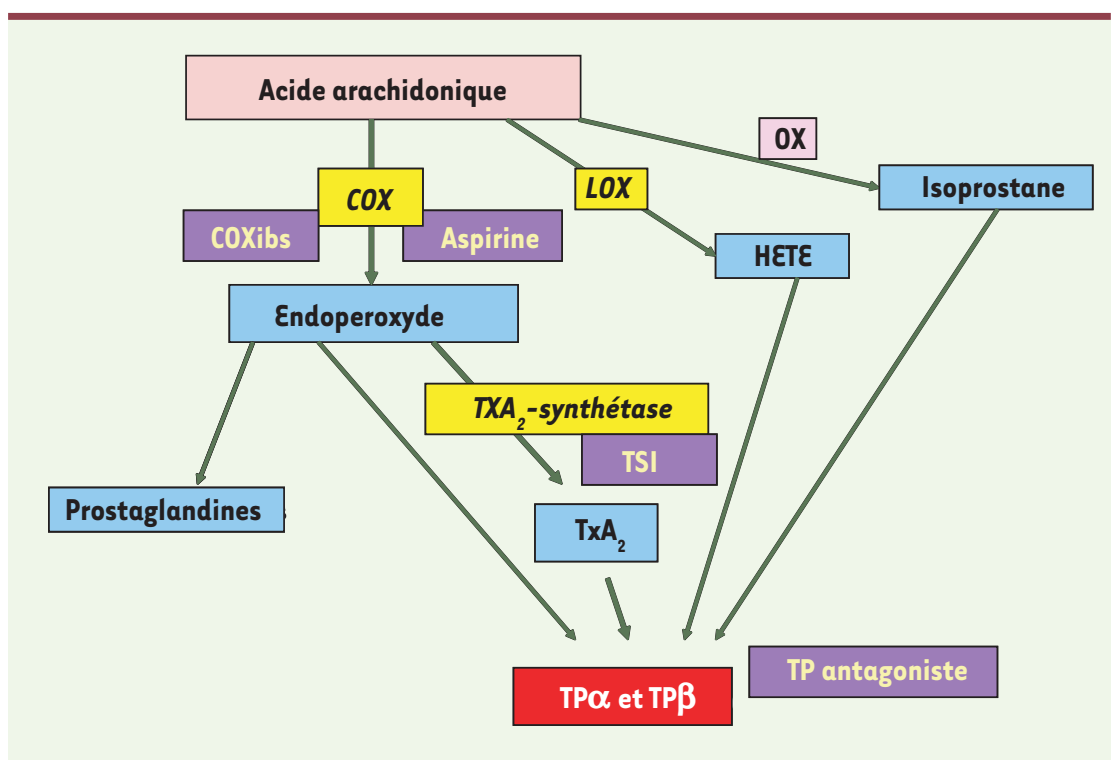

Figure 1. Des métabolites de l'acide arachidonique, formés par des voies différentes, peuvent activer les récepteurs TP. Le thromboxane $\mathrm{A}_{2}\left(\mathrm{TXA}_{2}\right)$ et son précurseur, l'endoperoxyde $\left(P \mathrm{PH}_{2}\right)$, sont formés par la voie de la cyclo-oxygénase ( $\left.C O X\right)$, tandis que les HETE (hydroxyeicosatetraenoic acids) sont formés par la voie de la lipoxygénase (LOX). Les isoprostanes sont, quant à elles, issues de l'oxydation non enzymatique (OX) de l'acide arachidonique. Les inhibiteurs de la COX, aspirine et Coxibs (inhibiteurs sélectifs de la (OX), préviennent la formation de l'endoperoxyde, tandis que les inhibiteurs de la $T X A_{2}$ synthétase (TSI) préviennent spécifiquement la synthèse du TXA 2 . Les antagonistes des récepteurs TP (TPr) bloquent de façon spécifique l'action du TXA ${ }_{2}$ sur son récepteur. dans les études cliniques $[8,9]$. Quant aux premiers antagonistes sélectifs des récepteurs TP (Figure 1) décrits, ils appartenaient à une série d'acides 13-azaprostanoïques bloquant les actions pharmacologiques de I'U46619, un agoniste puissant et sélectif des récepteurs TP [8]. Le premier antagoniste des récepteurs TP de structure non prostanoïde décrit a été le BM 13177, ou sulotroban; plusieurs autres antagonistes ont été développés depuis, comme le daltroban, le ramatroban et le seratrodast. Le terutroban, S 18886 (Figure 2), issu de la recherche du Groupe Servier, appartient à cette famille d'antagonistes des récepteurs TP.

L'Institut de Recherche Servier (IDRS) s'est engagé dans le domaine de la recherche sur la thrombose au début des années 90, avec pour objectif initial de rechercher un antagoniste des récepteurs TP. Le S 18204 a été découvert en 1993 [10,11], et ses propriétés antiplaquettaires et antivasoconstrictrices ont été étudiées: cette substance racémique est un antagoniste des récepteurs TP, puissant, sélectif, actif par voie orale; il possède une longue durée d'action, et diminue la micro-albuminurie chez le rat diabétique [12-14].

Ces résultats ont été confirmés avec le terutroban, son énantiomère actif, qui peut également démasquer les actions relaxantes puissantes des prostaglandines $P G I_{2}$ et $P_{G D}$ [15]. Le terutroban se lie de façon spécifique et durable aux récepteurs TP plaquettaires [16]. Une étude clinique a permis de montrer que la concentration plasmatique minimale nécessaire pour obtenir un effet anti-agrégant pendant 24 heures est obtenue dès une dose d'administration de $10 \mathrm{mg}$ [17]. Le terutroban, administré à cette dose à des patients coronariens déjà traités par l'aspirine, améliorait leur fonction endothéliale [18]; le dysfonctionnement endothélial chez ces patients semblait impliquer une production de métabolites de l'acide arachidonique (des isoprostanes) indépendante de la COX, et pouvant activer les récepteurs TP [18]. Une surproduction de substances vasoconstrictrices peut en effet provoquer une dysfonction endo-

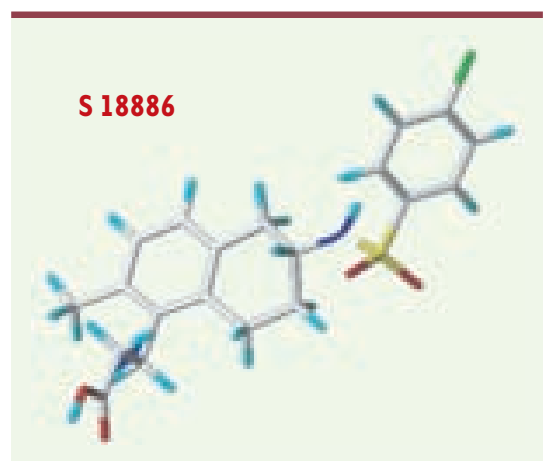

Figure 2. Structure du terutroban (S 18886). 
théliale, comme cela a été démontré au cours de l'hypertension [19]. Plusieurs études précliniques réalisées avec le terutroban ont montré qu'un agoniste des récepteurs TP, produit par l'activité de la COX-1 et libéré par l'endothélium, est à l'origine de ce dysfonctionnement chez le rat hypertendu SHR (spontaneously hypertensive rat) [20]. Enfin, l'activité antithrombotique du terutroban a été confirmée chez différents modèles animaux (cobaye, chien et porc) $[14,21,22]$.

L'ensemble de ces études témoigne que le terutroban, en inhibant les récepteurs TP, possède des effets antivasoconstricteurs et antithrombotiques puissants, compatibles avec le rôle reconnu de ces récepteurs vasculaires et plaquettaires. Mais le terutroban a encore d'autres actions...

\section{Rôle des récepteurs TP dans l’athérosclérose}

L'infarctus du myocarde, l'artériopathie des membres inférieurs et l'accident vasculaire cérébral sont largement liés à la formation de plaques d'athérosclérose dans les artères. Ces plaques, nées d'une activation des cellules endothéliales et mettant en jeu l'ensemble du processus d'inflammation, influent également sur l'activité vasomotrice des artères. L'observation de l'absence totale de relaxation dépendante de l'endothélium dans des artères athéromateuses prélevées chez des lapins hypercholestérolémiques [23, 24] a conduit à de nombreuses études sur le dysfonctionnement endothélial.

Il est probable que le manque d'intérêt pour le rôle du TXA 2 et des récepteurs TP dans le développement des plaques athéromateuses provient du peu de preuves cliniques ou expérimentales en faveur d'une action de l'aspirine, ou d'autres agents antiplaquettaires, sur l'athérosclérose [25]. De fait, une étude, seulement, a montré qu'un antagoniste des récepteurs TP, le daltroban, réduit le dépôt de cholestérol et la formation de plaques dans l'artère coronaire de lapins hypercholestérolémiques [26]. Avec l'équipe de Richard Cohen, nous avons débuté l'évaluation de l'effet du terutroban sur le développement de la maladie athéroscléreuse en 1998. En quoi l'inhibition des récepteurs TP serait-elle supérieure à une inhibition de la coX par l'aspirine?
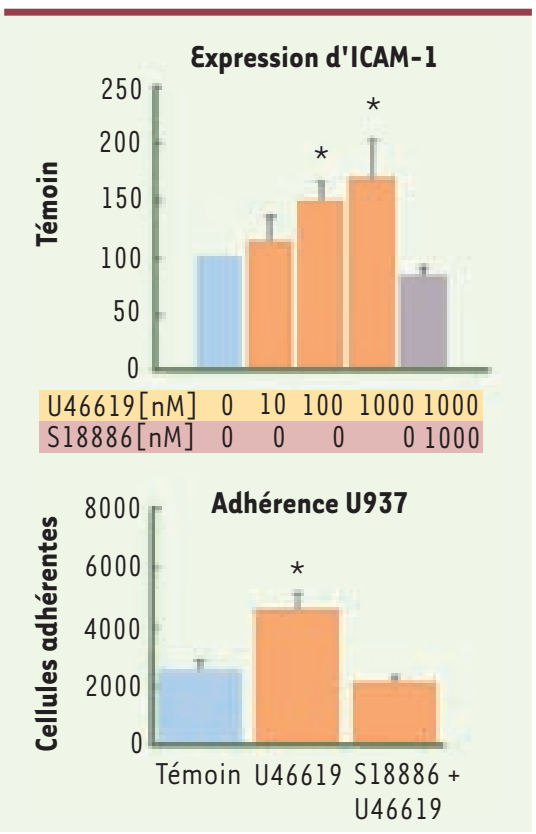

Figure 4. Le terutroban (S 18886) inhibe l'expression de l'ICAMI (en haut) et l'adhérence des monocytes (en bas) provoquées par l'agoniste des récepteurs TP, le U 46619. * Augmentation significative obtenue avec le U46619 ( $p<0,05)$ (d'après [25]). 
Notre hypothèse était la suivante: l'activation des récepteurs TP n'intervient pas seulement par le $\mathrm{TXA}_{2}$, mais également par d'autres prostaglandines $\left(\mathrm{PGH}_{2}, \mathrm{PGF}_{2} \alpha\right)$, ainsi que par des métabolites de l'acide arachidonique non dépendants de l'activité de la COX, tels que les acides hydroxy-eicosatétra-énoïques (HETE) [27] ou les isoprostanes [28] (Figure 1). Ces composés, dont la production est augmentée au cours de l'athérosclérose, pourraient accélérer la formation des plaques d'athérome par une activation des récepteurs TP non sensibles à l'aspirine, probablement des récepteurs TP endothéliaux d'isoforme TP $\beta$ [25].

Dans une première étude [25], des souris déficientes en apolipoprotéine $\varepsilon$ ( $A p o \varepsilon K O)$, qui développent des lésions athéromateuses dans la crosse de l'aorte, ont été traitées pendant 12 semaines avec le terutroban $(5 \mathrm{mg} / \mathrm{kg} / \mathrm{j})$, l'aspirine $(30 \mathrm{mg} / \mathrm{kg} / \mathrm{j})$ ou un placebo. Aucune modification du poids corporel ou de la cholestérolémie n'a été observée. Le terutroban réduisait la surface des lésions d'environ $25 \%$, tandis que l'aspirine était sans effet sur ce paramètre, malgré une forte diminution des concentrations sanguines de $\mathrm{TXA}_{2}$ (Figure 3 ): l'activation des récepteurs TP contribuait donc à la formation de lésions athéromateuses dans ce

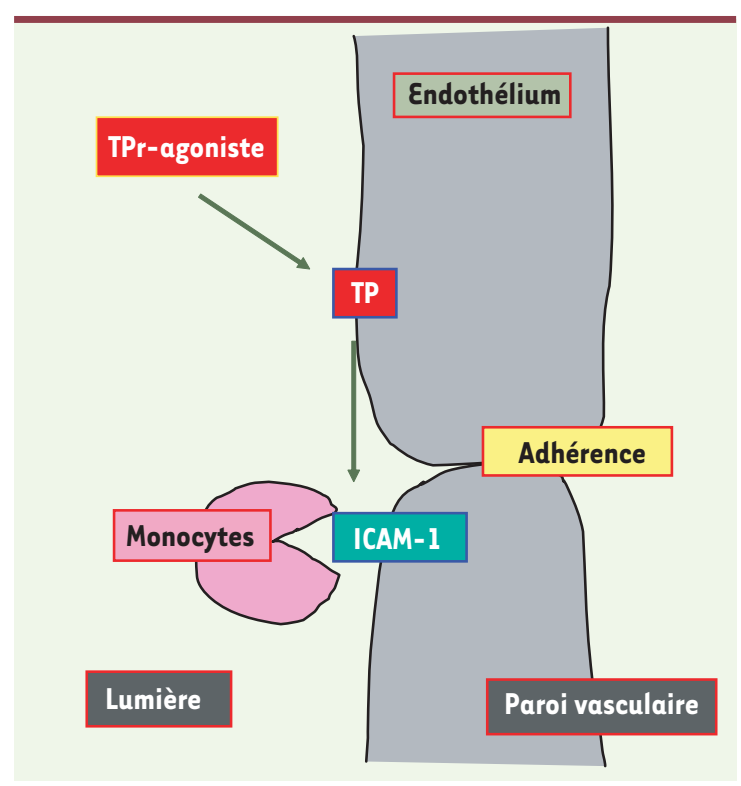

Figure 5. Effet de l'activation des récepteurs TP endothéliaux. Le TXA ${ }_{2}$ active le récepteur TP endothélial, ce qui résulte en une expression de molécules d'adhérence comme ICAMI, et à une adhérence des monocytes à la paroi vasculaire. modèle, mais sans implication du $\mathrm{TXA}_{2}$; en revanche, la production d'une isoprostane pouvait jouer un rôle. Le laboratoire de Richard Cohen a ensuite montré que le terutroban, mais pas l'aspirine, diminuait de $25 \%$ les concentrations d'ICAMl (intercellular adhesion molecule 1) soluble chez les souris ApoE KO [25] : l'effet du terutroban pouvait donc être lié à l'inhibition de l'expression de molécules d'adhérence (Figure 3), ce qui a été confirmé dans un modèle de cellules endothéliales ombilicales humaines (HUVEC) : I' agoniste des récepteurs TP, le U 46619, augmente l'expression de l'ICAM-1 à la surface de la membrane des HUVEC $[25,29]$, ainsi que l'adhérence de monocytes, ces effets étant totalement inhibés par le terutroban à la dose de $1 \mu M[25]$ (Figure 4).

À partir de ces données, nous avons formulé une hypothèse selon laquelle l'athérogenèse dépendrait, au moins en partie, de l'activation, indépendante du $T_{X A_{2}}$, de récepteurs endothéliaux; cette activation entraînerait l'expression de molécules d'adhérence et une infiltration subséquente de leucocytes inflammatoires pro-athérogènes dans la paroi artérielle (Figure 5). La suppression de l'expression des récepteurs TP (TP KO) dans un modèle de souris ApoE KO retarde l'athérogenèse, cet effet pouvant être attribué à l'inhibition de l'activité plaquettaire et des interactions entre leucocytes et cellules endothéliales [30].

Contrairement au traitement par l'aspirine, l'administration de terutroban à des souris ApoE KO rendues diabétiques permet d'obtenir, de façon très importante, une diminution de l'apparition des plaques d'athérome, sans pour autant influer sur les

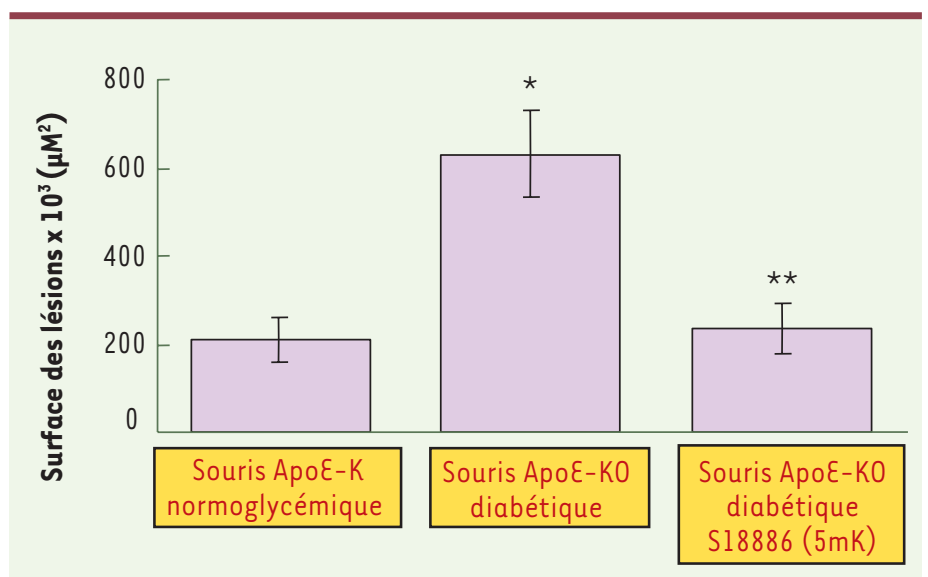

Figure 6. Le terutroban (S 18886) inhibe fortement les lésions athéromateuses dans l'aorte de souris déficientes en Apoz, rendues diabétiques. Les lésions aortiques sont plus importantes chez la souris Apoz diabétique que chez la souris Apoz. Le traitement avec le terutroban à la dose de $5 \mathrm{mg} / \mathrm{kg} / \mathrm{j}$ ( $5 \mathrm{mK}$ ) diminue fortement la formation de ces lésions. * Surface de lésions plus importante

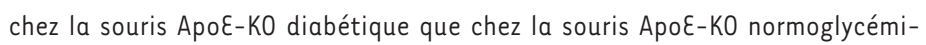
que $(p<0,05)$. ** Surface de lésions moins importante chez la souris Apo\&-KO diabétique traitée avec le $\mathrm{S} 18886$ que chez la souris Apo\&-KO diabétique non traitée $(p<0,05)$ (d'après [37]). 
paramètres métaboliques (Figure 6) [31]. Le dysfonctionnement endothélial observé chez ces souris est prévenu par le traitement avec le terutroban [31]; ces résultats concordent avec ceux d'une étude, réalisée chez le lapin hypercholestérolémique, ayant montré que le ramatroban améliore les réponses réduites à l'acétylcholine, probablement en inhibant l'action d'une isoprostane sur des récepteurs TP [32]. Enfin, l'étude de cellules endothéliales aortiques humaines démontre que la diminution de l'expression de la NO-synthétase endothéliale (eNOS) et l'augmentation de l'expression des molécules d'adhérence ICAMI et VCAM-1 (vascular cell adhesion molecule 1), provoquées par le U 46619, le TNF $\alpha$ ou une concentration de glucose élevée, n'apparaissent plus dans les cellules traitées par le terutroban [31].

Le travail initial de Cohen et de ses collaborateurs, montrant l'effet bénéfique du terutroban sur l'athérogenèse, a été confirmé dans plusieurs autres études [33-36]. Le rôle de la prostacycline dans les effets bénéfiques du terutroban a également été souligné chez les souris surexprimant le récepteur TP humain (TP $0 \varepsilon$ ), ainsi que chez les souris invalidées pour les récepteurs TP (TP KO) [34]. Quant aux résultats de l'étude d'Egan et de ses collaborateurs [33], ils montrent que les agonistes des récepteurs TP sont impliqués dans la genèse et la progression initiale de l'athérosclérose chez la souris Apobec-1/LDL receptor $K 0$, mais ne jouent pas de rôle important lorsque la maladie est établie dans ce modèle.

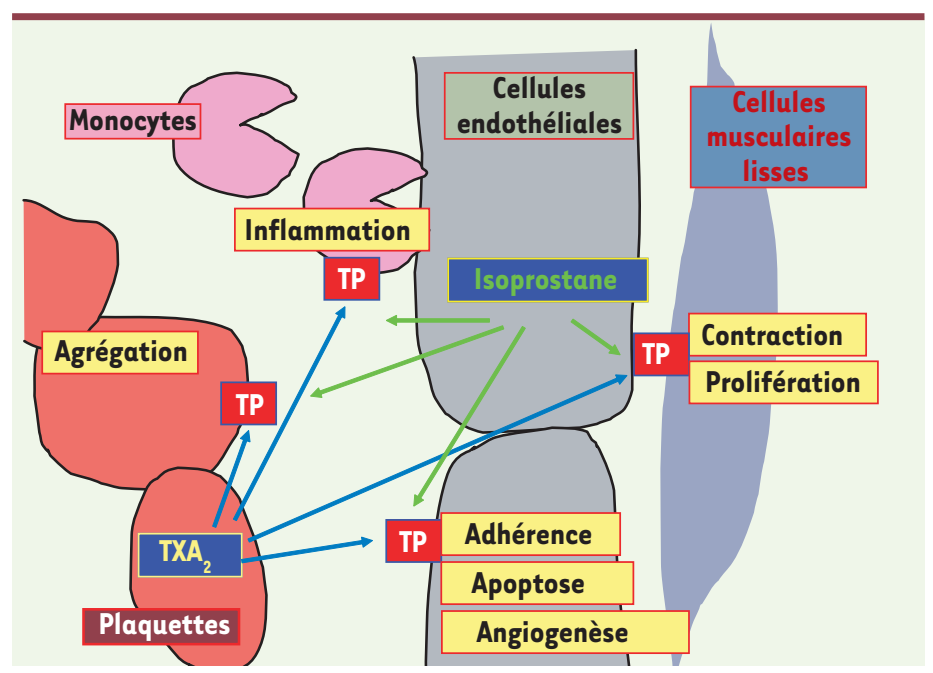

Figure 7. Localisation et activité des récepteurs TP vasculaires. L'activation des récepteurs TP présents sur les plaquettes provoque leur agrégation. L'activation des récepteurs TP monocytaires, encore mal étudiée, pourrait être impliquée dans l'inflammation. L'activation des récepteurs TP des cellules musculaires lisses provoque leur contraction et leur prolifération. L'activation des récepteurs TP présents sur les cellules endothéliales provoque l'expression de molécules d'adhérence, impliquant ces récepteurs dans l'athérosclérose; ces récepteurs TP endothéliaux semblent également être impliqués dans l'apoptose et l'angiogenèse.
L'implication des récepteurs TP dans d'autres modèles d'athérosclérose a également été étudiée avec le terutroban. Julie Campbell et ses collaborateurs ont examiné les effets du terutroban sur le développement des stries lipidiques et des lésions athéromateuses plus avancées dans un modèle d'athérosclérose et de resténose chez le lapin: le traitement réduisait les lésions artérielles, l'expression de I'ICAM-l et l'accumulation des macrophages après deux procédures d'introduction d'un ballonnet dans l'artère [35]. Les données de cette étude confirment les résultats initiaux de Richard Cohen et de ses collaborateurs, et $y$ ajoutent l'effet sur l'infiltration des macrophages, renforçant ainsi notre hypothèse. Worth et de ses collaborateurs sont arrivés à la conclusion que le terutroban peut favoriser la stabilité des plaques d'athérome en interférant avec le processus inflammatoire qui conduit ultimement à la rupture et à la thrombose [35]. L'étude de Juan Badimon et de ses collaborateurs a, quant à elle, montré que le terutroban peut provoquer la régression des plaques athéromateuses avancées et transformer ces lésions en un phénotype plus stable [36]. Ces résultats concordent avec ceux de l'équipe de Julie Campbell [35] et avec les résultats obtenus dans les modèles de souris [25,31], l'exception étant l'étude d'Egan et al. [33] qui suggèrent que le terutroban n'aurait pas d'impact sur les lésions avancées.

Les données scientifiques confirment généralement les observations initiales quant au rôle joué par l'activation des récepteurs TP dans l'athérogenèse et dans la progression de la maladie, par l'intermédiaire de l'adhérence cellulaire et de l'infiltration des monocytes/macrophages. Les récepteurs TP endothéliaux, contrairement aux récepteurs TP des plaquettes ou des cellules musculaires lisses, n'ont pas encore été étudiés en détail et leurs mécanismes doivent être éclaircis. Une première étude de Cohen et de ses collaborateurs indique que l'activation des récepteurs TP endothéliaux est impliquée dans les phénomènes d'oxydation présents dans la néphropathie diabétique [37].

Il semble évident que les antagonistes des récepteurs TP, en inhibant les récepteurs TP endothéliaux, possèdent des propriétés anti-athéromateuses puissantes, comme l'a montré la recherche menée avec le terutroban. 


\section{Conclusions}

La découverte du terutroban (S 18886) en 1993 a été à l'origine d'une série d'études mettant en évidence le rôle des récepteurs TP (les récepteurs du TXA ${ }_{2}$ ) dans le développement des lésions athéromateuses. L'activation des récepteurs TP joue un rôle dans la thrombose (agrégation plaquettaire par les récepteurs TP plaquettaires) et dans les vasospasmes (vasoconstriction par les récepteurs TP des cellules musculaires lisses). Nous savons maintenant que l'adhérence cellulaire et l'infiltration monocytaire sont également sous contrôle des récepteurs TP, localisés sur les cellules endothéliales et appartenant à l'isoforme TP $\beta$ (Figure 7). II convient de signaler que des récepteurs TP monocytaires ont également été décrits [38] (Figure 7), mais que leur rôle dans la physiologie et la physiopathologie est encore à déterminer.

L'activation des récepteurs TP peut conduire à la prolifération des cellules musculaires lisses [39] et être impliquée dans l'apoptose et dans l'angiogenèse [40]. Certains de ces effets peuvent aider à expliquer le rôle des récepteurs TP dans l'athérogenèse. Par ailleurs, les processus inflammatoires jouent un rôle essentiel dans le développement des maladies cardiovasculaires, et sont primordiaux dans l'athérogenèse. Des résultats récents plaident pour une activation des récepteurs TP endothéliaux, qui conduirait à l'expression des molécules d'adhérence et à l'infiltration des cellules inflammatoires (Figure 7) au cours de la formation des plaques. $\varepsilon$ n raison de sa capacité à inhiber ces récepteurs TP, le terutroban exerce donc un effet bénéfique protecteur en bloquant la genèse et la progression des lésions athéromateuses [41].

Les isoprostanes, métabolites de l'acide arachidonique résultant du stress oxydatif, pourraient intervenir dans l'activation des récepteurs TP sans intervention directe du TXA 2 [25, 31, 37]; ainsi, le lien entre les récepteurs TP, le stress oxydatif et l'athérosclérose semble évident [37]. On notera que le terutroban inhibe complètement l'action des isoprostanes, tandis que l'aspirine n'inhibe ni leur production, ni leur action. Les propriétés pro-athérogènes de l'isoprostane $\mathrm{F}_{2 \alpha}$-III ont récemment été rapportées dans deux modèles de souris, ApoE KO et $L D L r K O$, ces effets étant observés à une dose qui double la concentration plasmatique de l'isoprostane [42].

La triple activité antithrombotique, antivasoconstrictrice et anti-athéromateuse du terutroban, observée dans des études expérimentales précliniques, est con- fortée par les premiers résultats de développement clinique et positionne le terutroban comme un médicament innovant, au potentiel unique pour le traitement des maladies cardiovasculaires. $\diamond$

\section{SUMMARY}

Terutroban and endothelial TP receptors

in atherogenesis

Treatment of thrombotic diseases implicates the use of anti-platelet agents, anti-coagulants and pro-fibrinolytic substances. Amongst the anti-platelet drugs, aspirin occupies a unique position. As soon as it became evident that the major action of aspirin is indirect blockade, through inhibition of cyclooxygenase (COX), of the production of thromboxane $A_{2}\left(T X A_{2}\right)$, a powerful vasoconstrictor and platelet activator, research for new anti-thrombotics that interact more specifically with the production and/or the action of $T_{X A_{2}}$ was started. Terutroban (S 18886) is a selective antagonist of TP receptors, the receptors for $\mathrm{TXA}_{2}$, that are present on platelets and on vascular smooth muscle cells, but also on endothelial cells. The role played by the platelet and smooth muscle cell TP receptors in thrombotic disease is well known, and preclinical and clinical studies with terutroban have illustrated the powerful antithrombotic effects of this agent. The implication of endothelial TP receptors in the development of atherosclerotic disease has only been examined during the past five years and studies with terutroban have been crucial for understanding the role of these endothelial receptors in cardiovascular physiopathology. The goal of the present review is to discuss the arguments in favour of the hypothesis suggesting that activation of endothelial TP receptors, by causing expression of adhesion molecules, favours adhesion and infiltration of monocytes/macrophages in the arterial wall, thereby stimulating the development of atherosclerosis. The review will also highlight the important contribution of the studies performed with terutroban in this research area. The triple activity (anti-thrombotic, anti-vasoconstrictor, anti-atherosclerotic) observed with terutroban in preclinical studies, stressed by the first results in clinical development, places terutroban as an innovative drug with a unique potential for treatment of cardiovascular disorders. $\diamond$

\section{REMERCIEMENTS}

Je remercie mon ami et collègue Gilbert Lavielle qui, avec les membres de son équipe, a réussi la synthèse (complexe) du terutroban en 1993, et m'a aidé à rédiger le présent manuscrit. Mes remerciements aussi à l'équipe d'angiologie de I'IDRS, qui a contribué à l'exploration pharmacologique du terutroban. Un grand remerciement à mon ami Richard Cohen et à son équipe à Boston, pour un travail scientifique remarquable mettant en évidence les propriétés anti-athéromateuses du terutroban. Et je remercie aussi l'équipe de développement de Courbevoie pour les études de pharmacologie qu'ils ont fait réaliser avec le terutroban dans le domaine de l'athérosclérose. Mes remerciements à Mme Christine Lamarche-Arène pour l'aide rédactionnelle. Et, enfin, j'ai apprécié le soutien, les suggestions et les commentaires du Dr Emmanuel Canet, vice-président de la recherche Servier. 


\section{RÉFÉRENCES}

1. Hamberg M, Svennsson J, Samuelson B. Thromboxanes: a new group of biologically active compounds derived from prostaglandin endoperoxides. Proc Natl Acad Sci USA 1975 ; $72: 2994-8$.

2. Vane JR, Flower R, Botting RM. The mechanism of action of aspirin. In : Vane JR, Botting RM, eds. Aspirin and other salicylates. London: Chapman and Hall Medical, 1992 : 35-59.

3. Awtry EH, Loscalzo J. Aspirin. Circulation $2000 ; 101: 1206-18$.

4. Narumiya S, Sugimoto $Y$, Ushikubi F. Prostanoid receptors : structures, properties, and functions. Physiol Rev 1999 ; 79 : 1193-226.

5. Halushka PV. Thromboxane $A_{2}$ receptors : where have you gone? Prostagl Lipid Med 2000 ; $60: 175-89$.

6. Hirata $M$, Ushikubi F, Kakizuka $A$, et al. Two thromboxane $A_{2}$ receptor isoforms in human platelets. Opposite coupling to adenylyl cyclase with different sensitivities to Arg60 to Leu mutation. J Clin Invest 1996; 97 : 949-56.

7. Kinsella BT. Thromboxane $A_{2}$ signalling in humans : a "tail" of two receptors. Biochem Soc Trans $2001: 29: 641-54$.

8. Hall SE. Thromboxane $A_{2}$ receptor antagonists. Med Res Rev $1991 ; 11: 503-79$.

9. Dogné JM, Hanson J, de Leval X, et al. New developments on thromboxane modulators. Mini Rev Med Chem $2004 ; 4: 649-57$

10. Verbeuren TJ, Simonet S, Descombes JJ, et al. S 18204 : a new powerful TXA2-receptor antagonist with a long duration of action. Thromb Haemost $1995 ; 73: 1324$.

11. Cimetière B, Dubuffet T, Muller 0 , et al. Synthesis and biological evaluation of new tetrahydronaphthalene derivatives as thromboxane receptor antagonists. Bioorg Med Chem Lett $1998 ; 8: 1375-80$.

12. Verbeuren TJ, Vallez MO, Petit C, et al. Amelioration of albuminuria in the diabetic rat by $S$ 18204, an anti-thromboxane receptor antagonist. Circulation 1995; $92: 222$.

13. Simonet S, Descombes JJ, Vallez M0, et al. S 18886, a new thromboxane (TP)-receptor antagonist is the active isomer of $S 18204$ in all species, except in the guinea-pig. In : Sinzinger $\mathrm{H}$ et al., eds. Recent advances in prostaglandin, thromboxane and leukotriene research. New York: Plenum Press, 1998 : 173-6.

14. Vallez MO, Rupin A, De Nanteuil G, Verbeuren T. Synergistic antithrombotic effects of the TPreceptor antagonist $S 18886$ and the direct thrombin inhibitor S 31307 in a thrombosis model of the rat abdominal aorta. Thromb Haemost 2001 ; P1625.

15. Verbeuren T, Descombes JJ, Simonet S, et al. The TP-receptor antagonist S 18886 unmasks vascular relaxation and potentiates the anti-platelet action of PGD2. Thromb Haemost 1997 ; 693

16. Descombes JJ, Menant Y, Lavielle G, Verbeuren TJ. Binding properties of a potent, long acting new thromboxane receptor antagonist, [3H] S 18886, on human, rat and dog platelet membranes. BrJ Pharmacol 1997; 122 : 381P.

17. Gaussem P, Rény JL, Thalamas C, et al. The specific thromboxane receptor antagonist S 18886 : pharmacokinetic and pharmacodynamic studies. J Thromb Haemost 2005; 3: 1437-45.

18. Belhassen L, Pelle G, Dubois-Rande JL, Adnot S. Improved endothelial function by the thromboxane $A_{2}$ receptor antagonist $S 18886$ in patients with coronary artery disease treated with aspirin. J Am Coll Cardiol 2003; 41 : 1198-204.

19. Taddei S, Virdis A, Ghiadoni L, et al. Endothelial dysfunction in hypertension. J Cardiovasc Pharmacol $2001 ; 38$ : S11-4.

20. Yang D, Félétou $M$, Levens $N$, et al. A diffusible substance(s) mediates endotheliumdependent contractions in the aorta of SHR. Hypertension $2003 ; 41: 143-8$.

21. Maalej N, Osman HE, Shanmuganayagam D, et al. Antithrombotic properties of the thromboxane $A_{2}$ /prostaglandin $\mathrm{H}_{2}$ receptor antagonist $\mathrm{S} 18886$ on prevention of plateletdependent cyclic flow reductions in dogs. J Cardiovasc Pharmacol 2005 ; 45 : 389-95.

22. Osende JI, Shimbo D, Fuster V, et al. Antithrombotic effects of S 18886, a novel orally active thromboxane $A_{2}$ receptor antagonist. J Thromb Haemost $2004 ; 2$ : 492-8.

23. Verbeuren TJ, Jordaens FH, Zonnekeyn LL, et al. Effect of hypercholesterolemia on vascular reactivity in the rabbit. I. Endothelium-dependent and endothelium-independent contractions and relaxations in isolated arteries of control and hypercholesterolemic rabbits. Circ Res 1986 ; 58 : 552-64.

24. Verbeuren TJ. Endothelium and coronary atherosclerosis, an update. Coronary Artery Disease 1993 ; numéro spécial : $72-87$.

25. Cayatte AJ, Du Y, Oliver-Krasinski J, et al. The thromboxane-receptor antagonist, S 18886 but not aspirin inhibits atherogenesis in apolipoprotein $\varepsilon$ deficient mice : evidence that eicosanoids other than thromboxane contribute to atherosclerosis. Arterioscler Thromb Vasc Biol $2000 ; 20: 1724-8$

26. Osborne JA, Lefer AM. Cardioprotective actions of thromboxane receptor antagonism in ischemic atherosclerotic rabbits. Am J Physiol 1988 ; 255 : H318-24.

27. Van Diest M, Herman AG, Verbeuren TJ. Influence of hypercholesterolemia on the reactivity of isolated rabbit arteries to 15-lipoxygenase metabolites of arachidonic acid: comparison with platelet-derived agents and vasodilators. Prostaglandins Leukot Essent Fatty Acids 1996; $54: 135-45$.
28. Montuschi P, Barnes PJ, Roberts LJII. Isoprostanes : markers and mediators of oxidative stress. FASEB J $2004 ; 18: 1791-800$.

29. Ishizuka T, Suzuki K, Kawakami M, et al. Thromboxane $A_{2}$ receptor blockade suppresses intercellular adhesion molecule- 1 expression by stimulated vascular endothelial cells. Eur J Pharmacol 1996 $312: 367-77$.

30. Kobayashi T, Tahara Y, Matsumoto M, et al. Roles of thromboxane $A_{2}$ and prostacyclin in the development of atherosclerosis in apo\&-deficient mice. J Clin Invest 2004 ; 114 : 784-94.

31. Zuccollo A, Shi C, Mastroani R, et al. The thromboxane $A_{2}$ receptor antagonist, $S$ 18886, prevents enhanced atherogenesis caused by diabetes mellitus. Circulation $2005 ; 112: 3001-8$.

32. Ishizuka T, Matsui T, Kurita A. Ramatroban, a TP receptor antagonist, improves vascular responses to acetylcholine in hypercholesterolemic rabbits in vivo. Eur J Pharmacol $2003 ; 468: 27-35$.

33. Egan KM, Wang M, Lucitt MB, et al. Cyclooxygenases, thromboxane, and atherosclerosis: plaque destabilization by cyclooxygenase- 2 inhibition combined with thromboxane receptor antagonism. Circulation 2005 ; $111: 334-42$.

34. Cheng $Y$, Austin SC, Rocca B, et al. Role of prostacyclin in the cardiovascular response to thromboxane $A_{2}$ Science $2002 ; 296$ : 539-41.

35. Worth NF, Berry CL, Thomas AC, Campbell JH. S18886, a selective TP receptor antagonist, inhibits development of atherosclerosis in rabbits. Atherosclerosis $2005 ; 183: 65-73$.

36. Viles-Gonzalez JF, Fuster V, Corti R, et al. Atherosclerosis regression and TP receptor inhibition : effect of $S 18886$ on plaque size and composition. A magnetic resonance imaging study. Eur Heart J 2005 ; 26 : 1557-61.

37. Xu S, Maitland KA, Jiang B, et al. The thromboxane receptor antagonist S18886 attenuates renal oxidant stress and proteinuria in diabetic Apo $\varepsilon$-deficient mice. Diabetes $2006 ; 55: 110-9$.

38. Simmons TR, Cook JA, Moore JN, Halushka PV. Thromboxane $A_{2}$ receptors in equine monocytes: identification of a new subclass of $\mathrm{TXA}_{2}$ receptors. J Leukoc Biol 1993 ; 53 : 173-8.

39. Nagata $T$, Uehara $Y$, Numabe $A$, et al. Regulatory effect of thromboxane $\mathrm{A}_{2}$ on proliferation of vascular smooth muscle cells from rats. Am J Physiol $1992 ; 263$ : H1331-8.

40. Gao Y, Yokota R, Tang $S$, et al. Reversal of angiogenesis in vitro, induction of apoptosis, and inhibition of Akt phosphorylation in endothelial cells by thromboxane $A_{2}$. Circ Res $2000 ; 87: 739-45$.

41. Verbeuren TJ, Cohen RA. Role of TP-receptors in atherosclerosis and vascular endothelial dysfunction. In: Abstracts of the Symposium on endothelial factors and coronary disease. Hong Kong, China, $2004: 22$ (abstract).

42. Tang $M$, Cyrus $T$, Yao $Y$, et al. Involvement of thromboxane receptor in the proatherogenic effect of isoprostane F2a-III. Evidence from apolipoprotein $\varepsilon$ - and LDL receptor-deficient mice. Circulation 2005; $112: 2867-74$.
TIRÉS À PART

T.J. Verbeuren 